\title{
Administration routes affect thrombolytic effect of catheter-directed thrombolysis with pro-urokinase in treating deep vein thrombosis
}

\author{
Meng Liu ${ }^{1,2}$, Fuxian Zhang ${ }^{1}$ \\ ${ }^{1}$ Department of Vascular Surgery, Beijing Shijitan Hospital, Capital Medical University, Beijing 100038, China; ${ }^{2}$ Department of Vascular Surgery, \\ Tianjin Hospital, Tianjin 300211, China \\ Contributions: (I) Conception and design: All authors; (II) Administrative support: F Zhang; (III) Provision of study materials or patients: F Zhang; (IV) \\ Collection and assembly of data: M Liu; (V) Data analysis and interpretation: All authors; (VI) Manuscript writing: All authors; (VII) Final approval \\ of manuscript: All authors. \\ Correspondence to: Fuxian Zhang. Department of Vascular Surgery, Beijing Shijitan Hospital, Capital Medical University, Tieyi Road No.10, \\ Yangfangdian, Haidian District, Beijing 100038, China. Email: fuxian_zhang@yeah.net.
}

Background: Deep venous thrombosis (DVT) is a common disorder with a significant mortality rate. Catheter-directed thrombolysis (CDT) is a preferred treatment for DVT patients. The aim of this study is to evaluate the thrombolytic effect of CDT with pro-urokinase (pro-UK) in different administration routes.

Methods: An inferior vena cava (IVC) model was induced in $36 \mathrm{New}$ Zealand white rabbits by catheter injury and placement of IVC filter. The animals were divided into 6 groups with 6 rabbits in each group: group A: systemic thrombolysis with intravenous injection; group B: CDT with pump infusion; group C: CDT with pulse injection; group D: CDT with combination of pump infusion and pulse injection; group E: catheter-directed aspiration thrombectomy; group F: mechanical thrombectomy. The thrombolytic effects were evaluated by color Doppler ultrasound and blood measurement of D-dimer and fibrinogen.

Results: The minimum effective concentration of pro-UK solution was identified. Two groups (group A and B) did not achieve successful thrombolysis. Among all catheter-directed administration groups, the rabbit groups that received pro-UK therapy through catheter-directed thrombolytic pump infusion combined with pulse injection (group D) showed the best thrombolysis effect. In contrast, catheter-directed aspiration thrombectomy (group E) is effective but not safe.

Conclusions: CDT with combination of pump infusion and pulse injection of pro-UK safely and significantly improves thrombolysis procedure in rabbits, suggesting potential benefits from the use of CDT in proximal DVT.

Keywords: Deep venous thrombosis (DVT); catheter-directed thrombolysis (CDT); pro-urokinase (pro-UK)

Submitted Mar 26, 2018. Accepted for publication Jun 12, 2018.

doi: $10.21037 /$ atm.2018.08.01

View this article at: http://dx.doi.org/10.21037/atm.2018.08.01

\section{Introduction}

Deep venous thrombosis (DVT), also called venous thrombosis, is characterized by the formation of blood clots (thrombi) in the deep veins $(1,2)$. DVT is a severe clinical entity that notably occurs in the lower limbs $(80-95 \%$ of cases). Over $20 \%$ of proximal DVT patients have symptomatic pulmonary embolism (PE), whereas about $50 \%$ have an associated asymptomatic PE noted with a lung scan, which together dramatically reduces short- and long-term survival among patients (3). The incidence rate of isolated DVT (without PE), and of PE (with or without DVT), range from 45 to 117 and 29 to 78 per 100,000 people yearly, respectively $(4,5)$. Given DVT is relatively common and associated with significant morbidity and mortality, significant progress has 

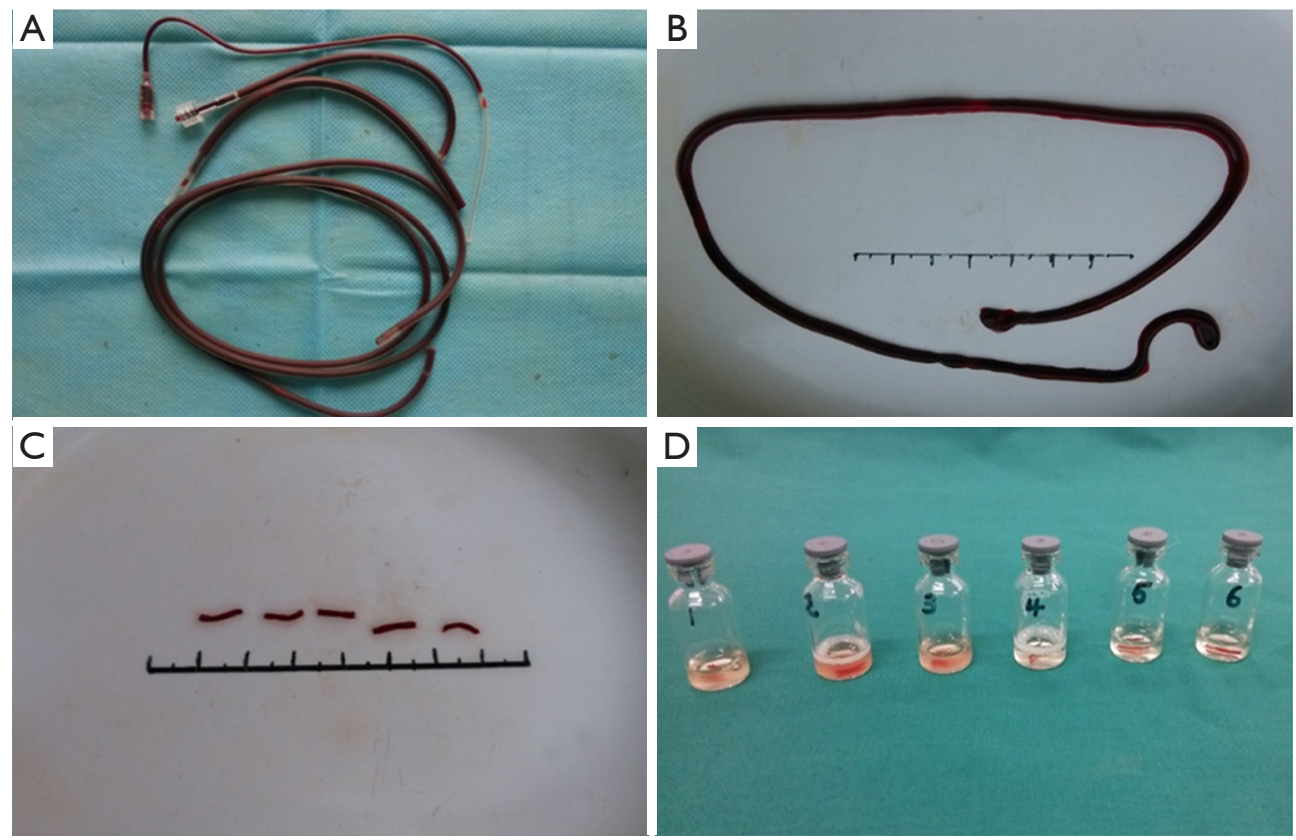

Figure 1 Thrombus formation and thrombolysis testing. (A) Thrombus was formed in infusion catheter in vitro; (B) cylindrical thrombus was pushed out by syringe; (C) 1-cm long thrombus was prepared; (D) thrombolysis testing in six bottles with difference concentration of pro-UK. Bottle \#1 with $0.25 \mathrm{mg} / \mathrm{mL}$ pro-UK showed the best thrombolysis efficacy. pro-UK, pro-urokinase.

been made in order to develop new therapies to control DVT and reduce immediate complications (6).

Anticoagulants are transitional drugs of choice for the treatment of both DVT and PE (7). Initial and longterm anticoagulation therapy remains the mainstay of medical therapy for DVT patients. New classes of oral anticoagulants such as Rivaroxaban and Dabigatran have proven to be highly selective at dissolving thromboses as well as reducing the incidence of post thrombotic syndrome (PTS) $(8,9)$. Despite advances in anticoagulant management with DVT patients, limitations to their use still exist, leading to the investigation of alternative strategies.

Emerging evidence showed strong clinical tendency of catheter-based recanalization therapies (CDT) with or without other endovascular methods in DVT patients (10). Previous studies have demonstrated that early vein recanalization reduced the incidence of post-thrombotic syndrome (PTS) which is a frequent complication of DVT $(11,12)$. Whereas, in the recent trial published in New England Fournal of Medicine, catheter-directed thrombolysis (CDT) failed to be confirmed to decrease the risk of the PTS due to larger size, different geographic and demographic scope as well as its greater use of mechanical therapies. Instead, the addition of pharmacomechanical CDT did result in a higher risk of major bleeding (13). No study, nevertheless, has explored whether the administration route of pro-urokinase (pro-UK) affects its effectiveness and safety. Here we used a rabbit inferior vena cava (IVC) thrombus model to test the safety and efficacy of human recombinant pro-UK therapy in different administration routes including CDT.

\section{Methods}

\section{The determination of the pro-UK dosage}

In order to optimize the working concentration of pro-UK, six 1-mm length cylindrical thrombi (Figure 1A,B,C) were generated and placed in six $5 \mathrm{~mL}$ glass bottles with $1 \mathrm{~mL}$ pro-UK solution at different concentrations $(0.25,1.0,0.5$, $0.125,0.0625,0 \mathrm{mg} / \mathrm{mL}$ ) (Figure 1D).

Changes of in vivo thrombolysis were assessed in vivo by $60-\mathrm{MHz}$ ultrasound imaging every $30 \mathrm{~min}$ after proUK treatment. The ultrasound criteria for a successful thrombolysis are: (I) the wall of the IVC at the location of the previous thrombus is closed by transducer, (II) the colorful signal of circulating heart blood flow is detected in the IVC at the location of the previous thrombus, and (III) the colorful signal of circulating heart blood flow is detected 

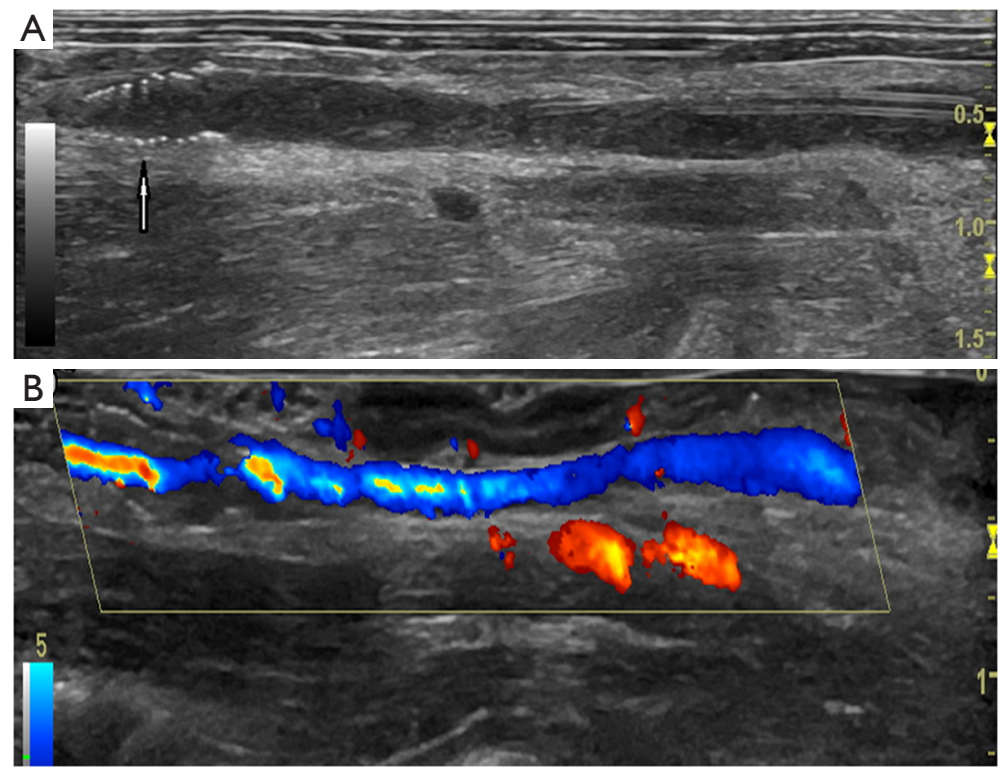

Figure 2 The color Doppler ultrasound images. Representative images showing changes before (A) and after (B) treatment with pro-UK. The thrombus is indicated by yellow arrow. pro-UK, pro-urokinase.

in the filter (Figure 2).

Six venous blood samples were collected at different times: the 3rd day after modeling; as well as 30, 60, 120, and 180 minutes after the thrombolysis therapy. Levels of D-dimer and fibrinogen in the blood were measured using ELISA kits (Abbexa, UK).

This study was performed in accordance with the National Institutes of Health guidelines for the care and use of laboratory animals. All procedures including operation and treatments on animals were approved by the Animal Care and Use Committee at Capital Medical University.

\section{Homemade IVC filter and infusion catheter}

The IVC filter used in the IVC thrombosis model was selfmade (Figure $3 A$ ). It was generated by twining a $0.17 \mathrm{~mm}$ diameter stainless steel wire into a 10 -circle, tower-shaped spring ring to induce thrombosis and to intercept the thrombus in the process of thrombolysis. The tip diameter of the filter was $1.5 \mathrm{~mm}$ and the bottom diameter was $4.0 \mathrm{~mm}$. The filter delivery system was self-made as well (Figure 3B). The delivery sheath was a $15 \mathrm{~cm}$ long plastic pipe with an external diameter of $4.5 \mathrm{~mm}$ and an internal diameter of $4.0 \mathrm{~mm}$. One end of the pipe was cut at a 40 -degree slope. The push cable was a 20 -cm long stainlesssteel wire with a $3.5-\mathrm{mm}$ diameter. The filter was carried into the sheath from the sloped end of the pipe with the tip in first. The catheter was made by puncturing the wall of an epidural catheter, used for anesthesia, every $0.5 \mathrm{~cm}$ by using a $1 \mathrm{~mL}$ syringe needle which was $5 \mathrm{~cm}$ in length (Figure 3C).

\section{Rabbit IVC thrombus model and treatments}

In total 36 healthy New Zealand white rabbits (Beijing Xinglong Laboratory Animal Technology Co., Ltd) at 5 months old were used. Induction of venous thrombosis in rabbits was performed by a transient ligation of the IVC for 1 hour. When the rabbits were under anesthesia with $3 \%$ sodium pentobarbital (Merck, Germany), a midline incision (about $8 \mathrm{~cm}$ in length) was made to expose the IVC. All the branches of the IVC were ligated with 8-0 silk threads and were artificially blocked for about 1 hour. The filter conveying system was inserted to perform ligation, followed by filter release and suture of the vascular wall through interruption with 6-0 Nylon. After the injury, slow blood flow and thrombosis formation below the filter could be detected by B-ultrasound. During recovery, the rabbits were given a dose of 400,000 $\mathrm{U}$ penicillin via intramuscular injection to prevent infection.

The procedure for thrombolysis is as follows: each experimental animal was given low-molecular-weight heparin calcium by subcutaneous injection at a dose of $200 \mathrm{U} / \mathrm{kg}$. All rabbits received pro-UK at $1 \mathrm{mg} / \mathrm{kg}$ with $0.25 \mathrm{mg} / \mathrm{mL}$ in 6 different types of routines. For systemic 

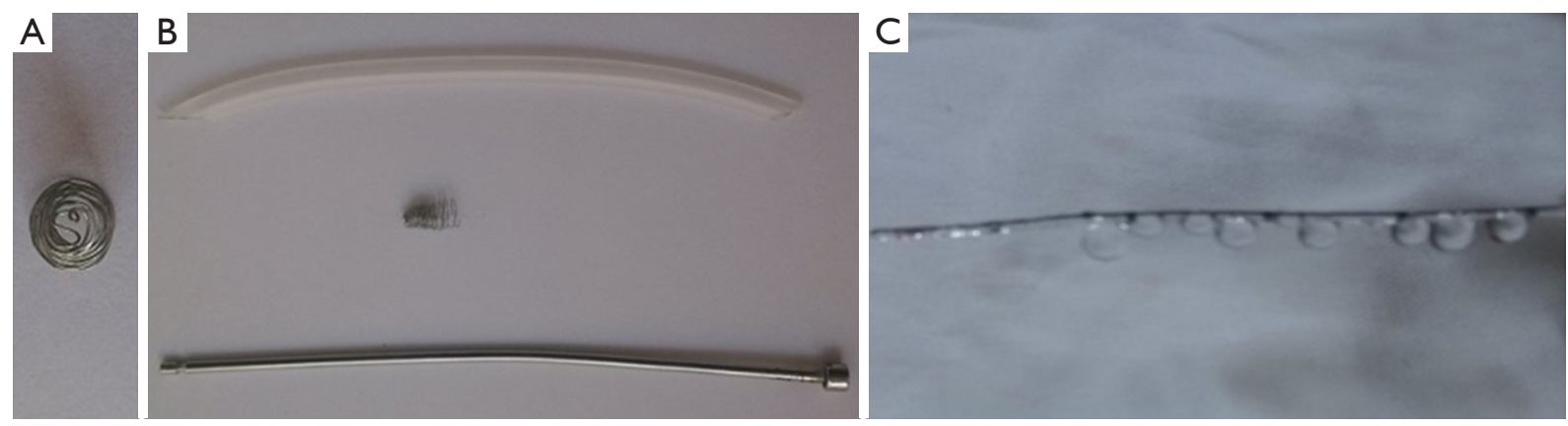

Figure 3 Homemade IVC filter and infusion catheter. (A) The self-designed IVC filter and (B) its conveying system; (C) self-designed infusion catheter. IVC, inferior vena cava.

thrombolysis with intravenous injection (group A), two-fifths of the total dose of pro-UK was dissolved in $0.6 \mathrm{~mL} \mathrm{NS}$ and then injected intravenously within 3 minutes. The rest was dissolved in $5.4 \mathrm{~mL} \mathrm{NS}$ and given by microinfusion pump via the auricular vein within 30 minutes. For CDT with pump infusion (group B) and CDT with pulse injection (group C), the infusion catheter was placed through the common femoral vein at $0.5 \mathrm{~cm}$ below the filter. The catheter was either connected to a microinfusion pump or a $1 \mathrm{~mL}$ syringe. Similarly, in the CDT with combination of pump infusion and pulse injection group (group D), the catheter was connected to a microinfusion pump to deliver half of the pro-UK and the rest was delivered by syringe. For catheterdirected aspiration thrombectomy (group E), a laparotomy surgery was performed under anesthesia to produce a closed lumen and block the IVC. In the mechanical thrombectomy group (group F), equal doses of NS, instead of pro-UK, were followed by aspiration thrombectomy repeated 3 times every 5 minutes. The dosage and time used in thrombolysis of each group were recorded.

\section{Statistical analysis}

Statistical analyses were performed using SPSS software (Version 19.0, United Kingdom). Data are given as mean $\pm \mathrm{SD}$ (standard deviation). One-way analysis of variance (ANOVA) with Dunnett Multiple Comparison test was used to determine the differences between groups. Statistical significance was defined as $\mathrm{P}$ value $<0.05$.

\section{Results}

\section{Determination of effective concentration of pro-UK}

Changes of thrombus volume and solution color were recorded for 1 hour. The minimum effective concentration of pro-UK solution was identified. Significant changes were observed in bottle \#1 30 minutes after pro-UK was given (Figure 1D). Therefore, a $0.25 \mathrm{mg} / \mathrm{mL}$ concentration was considered the effective concentration in our study.

\section{Determination of thrombus formation in rabbit models and the thrombolysis time}

One day post implant of the IVC filter, the thrombus forming was monitored using ultrasound imaging (Figure 4A). Typically, the thrombi that were formed resembled a whiteheaded, red-tailed thrombus. Our pilot experiment and previous report showed that the thrombus model became stable 3-7 days after IVD filter implant. In our study, the thrombus formed at day 3 and was stable at day 7 .

\section{Thrombolytic efficacy of pro-UK}

All rabbits with IVC thrombus were alive after the pro-UK treatments. No significant difference in body weight was observed (Table S1). Thrombi were completely dissolved in all 4 CDT rabbit groups (groups B-E). The required duration of thrombolysis therapy to achieve dissolution of the thrombus were recorded (Figure 4A). Neither systemic thrombolysis (group A) nor mechanical thrombectomy (group F) resulted in successful thrombolysis. Among all catheter-directed administration groups, the rabbits that received catheter-directed aspiration thrombectomy (group E) showed the best thrombolysis effect (Figure 4A).

Next, we sought to assess the effective dose of pro-UK in different groups. The total dose used in group E was $1.66 \mathrm{mg}$, which was significantly lower than other groups (Figure 4B). Given the duration required for thrombolysis, 

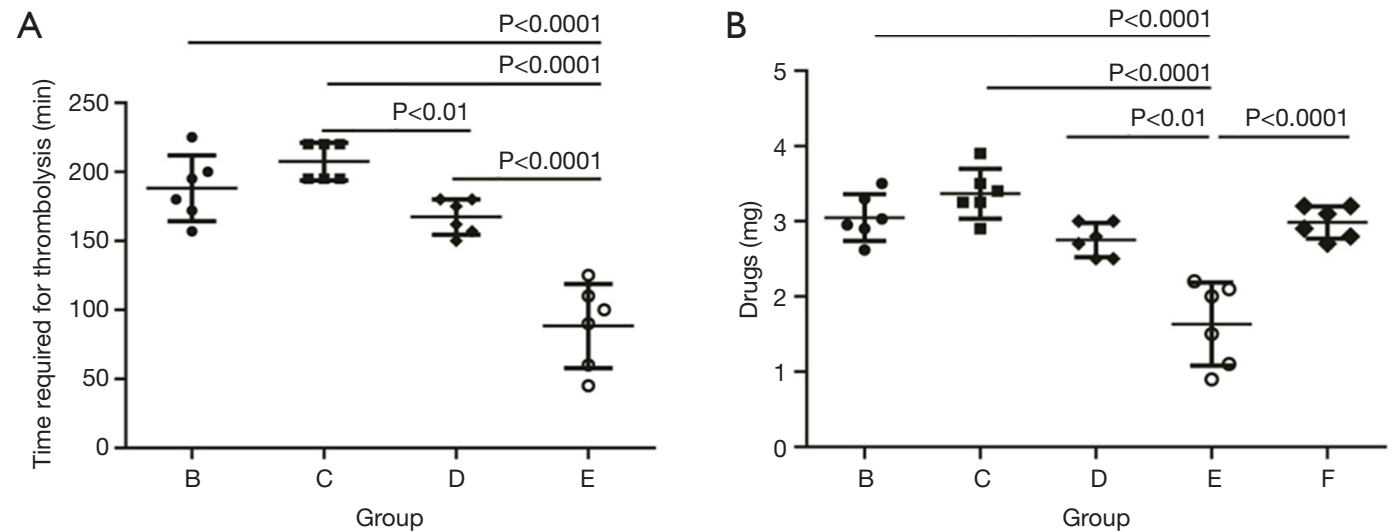

Figure 4 Thrombolytic efficacy of pro-UK administrated in various routines. Duration of thrombolysis therapy (A) and total amount of pro-UK (B) required in four CDT therapy groups. Group B: CDT with pump infusion; group C: CDT with pulse injection; group D: CDT with combination of pump infusion and pulse injection; group E: catheter-directed aspiration thrombectomy; group F: mechanical thrombectomy. pro-UK, pro-urokinase; CDT, catheter-directed thrombolysis.

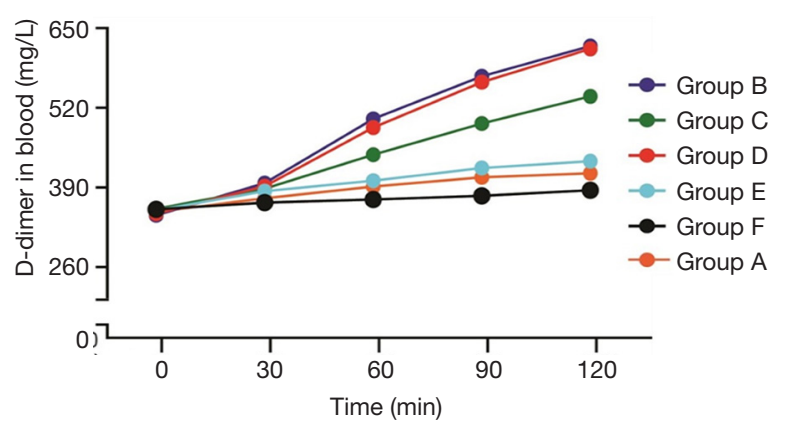

Figure 5 Blood D-dimer levels as measured during the thrombolysis. D-dimer levels in all 6 experimental groups were measured in 5 time points after thrombolysis. Group A: systemic thrombolysis with intravenous injection; group B: CDT with pump infusion; group C: CDT with pulse injection; group D: CDT with combination of pump infusion and pulse injection; group $\mathrm{E}$ : catheter-directed aspiration thrombectomy; group F: mechanical thrombectomy. CDT, catheter-directed thrombolysis.

we concluded both catheter-directed aspiration thrombectomy (group E), and catheter-directed thrombolytic pump infusion combined with pulse injection (group D) indicate the most efficient administration options for pro-UK mediated thrombolysis. However, in addition to effective and efficient thrombolysis, massive abdominal hemorrhage was observed in two out of six (1/3) rabbits in group E, while no major bleeding events was found in the other CDT groups (group B-D).

\section{Comparison of biochemical parameters}

Blood D-dimer levels can be used as an indicator of thrombolysis since it is released into the blood when fibrinolysis dissolves $(14,15)$. As shown in Figure 5 and Table 1, group B and group D exhibited the highest blood D-dimer levels while no significant difference was observed between these two groups. The blood D-dimer level in group $\mathrm{C}$ is also higher than the other three groups (group A, E, F). The detailed values of D-dimer levels are shown in Table 1 and the estimated levels of blood fibrinogen are shown in Table 2. We detected no significant difference in fibrinogen levels among the groups during thrombolysis.

\section{Discussion}

This study aimed to evaluate the therapeutic effect of pro$\mathrm{UK}$ in various administration routes. We demonstrated that catheter-directed pro-UK therapy is of significant benefit compared to systemic infusion of pro-UK or mechanical thrombectomy. We identified the optimized administration concentration and duration for pro-UK therapy.

Pro-UK is a precursor of urokinase, which is an enzyme that catalyzes the conversion of plasminogen to plasmin, thus stimulating fibrinolysis and depredating thrombi. The beneficial effect of pro-UK was originally shown in patients with myocardial infarction (16). Pro-UK is also approved for use in selected patients with ischemic stroke, but there is an expectation that it should benefit a much wider variety 
Table 1 Plasma levels of D-dimer in 6 experimental groups

\begin{tabular}{|c|c|c|c|c|c|}
\hline Group & \multicolumn{5}{|c|}{ Time points during the thrombolysis $(\mu \mathrm{g} / \mathrm{L})$} \\
\hline A & $335.0(43.9)$ & $358.9(56.0)$ & $379.4(58.7)$ & $396.8(60.1)$ & $402.8(60.0)$ \\
\hline $\mathrm{B}$ & 329.1 (39.6) & $385.3(41.4)$ & $498.1^{\#}(38.7)$ & $573.0^{\&}(34.9)$ & $625.9^{\&}(57.7)$ \\
\hline $\mathrm{C}$ & $340.4(46.1)$ & $375.4(44.8)$ & $435.4(53.1)$ & $489.9^{*}(45.9)$ & $537.7^{\#}(39.9)$ \\
\hline$E$ & $339.6(58.9)$ & $371.5(58.3)$ & $389.7(57.7)$ & $411.7(62.1)$ & $424.1(58.8)$ \\
\hline $\mathrm{F}$ & $339.5(49.2)$ & $351.3(44.6)$ & $356.5(46.9)$ & $363.3(48.0)$ & $373.0(48.4)$ \\
\hline
\end{tabular}

D-dimer levels $(\mu \mathrm{g} / \mathrm{L})$ are given as mean $(\mathrm{SD})$. Statistical significance: ${ }^{\star} \mathrm{P}<0.05,{ }^{\#} \mathrm{P}<0.01,{ }^{\&} \mathrm{P}<0.001$ when compared to group $\mathrm{A}$. Group A: systemic thrombolysis with intravenous injection; group B: CDT with pump infusion; group C: CDT with pulse injection; group D: CDT with combination of pump infusion and pulse injection; group E: catheter-directed aspiration thrombectomy; group F: mechanical thrombectomy. CDT, catheter-directed thrombolysis.

Table 2 Plasma levels of fibrinogen in 6 experimental groups

\begin{tabular}{|c|c|c|c|c|c|}
\hline Group & \multicolumn{5}{|c|}{ Time points during the thrombolysis $(\mathrm{g} / \mathrm{L})$} \\
\hline$A$ & $2.50(0.26)$ & $2.48(0.23)$ & $2.51(0.18)$ & $2.51(0.22)$ & $2.52(0.20)$ \\
\hline $\mathrm{B}$ & $2.53(0.17)$ & $2.54(0.09)$ & $2.54(0.10)$ & $2.50(0.13)$ & $2.52(0.21)$ \\
\hline $\mathrm{C}$ & $2.54(0.24)$ & $2.53(0.26)$ & $2.52(0.21)$ & $2.54(0.22)$ & $2.51(0.13)$ \\
\hline$E$ & $2.50(0.29)$ & $2.51(0.24)$ & $2.53(0.29)$ & $2.54(0.29)$ & $2.52(0.28)$ \\
\hline $\mathrm{F}$ & $2.44(0.26)$ & $2.44(0.24)$ & $2.46(0.23)$ & $2.46(0.23)$ & $2.47(0.23)$ \\
\hline
\end{tabular}

Fibrinogen levels (g/L) are given as mean (SD). Group A: systemic thrombolysis with intravenous injection; group B: CDT with pump infusion; group C: CDT with pulse injection; group D: CDT with combination of pump infusion and pulse injection; group E: catheterdirected aspiration thrombectomy; group F: mechanical thrombectomy. CDT, catheter-directed thrombolysis.

of patients (17). A number of trials are underway to assess and identify them. There are a few studies that investigated the potential of pro-UK being used as a new medicine for dissolving thrombi (18). However, whether it could be used to treat patients with DVT remains unknown. Moreover, it is rarely studied which administration route provides the best therapeutic potential in thrombolysis therapy. Our data demonstrated that catheter-directed therapy with pro-UK was significantly more efficient than pro-UK administered via intravenous infusion. The following reasons may explain the advantage of catheter-directed therapy: (I) the surface of the thrombi in contact with the drug in systemic thrombolysis is much smaller than catheter-directed therapy, and (II) catheter-directed administration increases local drug concentration and maintains high pro-UK activity.

CDT has been considered the basic treatment for DVT patients for decades, especially for patients with iliofemoral DVT, due to poor spontaneous recanalization in this segment (19). Our data is in agreement with the wellestablished understanding that CDT should minimize the risk of bleeding and $\mathrm{PE}$ for patients with contraindications to systemic thrombolysis (20). Our study also revealed that, although various CDT techniques and protocols exist, the safest and most efficient method is low-dose $(0.25 \mathrm{mg} / \mathrm{mL})$ catheter-directed thrombolytic pump infusion combined with pulse injection. Using pulse injection, pro-UK could physically break the thrombus due to fluid mechanics, thus reducing the size of the thrombus and increasing the thrombus drug contact area. On the other hand, the 
combination of pump infusion of pro-UK would further strengthen the thrombolysis process. Although in our study the most efficient method is catheter-directed aspiration thrombectomy, we do not recommend this option since the mechanical force may lead to serious vascular endothelial damage.

As is consistent with previous studies in rabbits and humans, our data indicated D-dimer is a possible blood marker for thromboembolic events (21-23). The levels of D-dimer continually increased during thrombolysis and were positively correlated with the extent of thrombolysis. We failed to detect a significant increase of D-dimer in group E (catheter-directed aspiration thrombectomy), which is likely because the lysate from the thrombolysis was pumped out and removed from the blood circulation. No significant difference in fibrinogen levels among all groups during thrombolysis was found, indicating the high selectivity of pro-UK with little side effects with respect to serum fibrinogen and bleeding.

\section{Limitation of the study}

In this study, healthy rabbits were used to assess the thrombolytic effect of pro-UK in DVT rabbits. DVT patients, however, normally have complications such as hypertension, diabetes, and hypercholesterolemia. Moreover, the sample size in each group was relatively small and needs further confirmation. Another limitation is due to technology. The interval period in the CDT-mediated pulse injection group was $15 \mathrm{~min}$, while the CDT-mediated interchange of the pulse injection was $30 \mathrm{~min}$, which may have led to a technology bias when comparing these two groups side by side.

\section{Conclusions}

Altogether, our results showed that CDT, with a combination of pump infusion and pulse injection of pro-UK, safely and significantly improves thrombolysis procedure in rabbits. Thus, this method gives a new enlightenment of how to better treat DVT patients in clinical practice. For more details, further studies are warranted hereafter.

\section{Acknowledgements}

This study was financially supported by Ph.D. Student Grant from Beijing Shijitan Hospital, Capital Medical
University, Beijing.

\section{Footnote}

Conflicts of Interest: The authors have no conflicts of interest to declare.

Ethical Statement: All procedures including operation and treatments on animals were approved by the Animal Care and Use Committee at Capital Medical University (No. 11401400000617).

\section{References}

1. Saleh J, El-Othmani MM, Saleh KJ. Deep Vein Thrombosis and Pulmonary Embolism Considerations in Orthopedic Surgery. Orthop Clin North Am 2017;48:127-35.

2. Streiff MB, Agnelli G, Connors JM, et al. Guidance for the treatment of deep vein thrombosis and pulmonary embolism. J Thromb Thrombolysis 2016;41:32-67.

3. Bruni-Fitzgerald KR. Venous thromboembolism: An overview. J Vasc Nurs 2015;33:95-9.

4. Heit JA. Epidemiology of venous thromboembolism. Nat Rev Cardiol 2015;12:464-74.

5. Thaler J, Pabinger I, Ay C. Anticoagulant Treatment of Deep Vein Thrombosis and Pulmonary Embolism: The Present State of the Art. Front Cardiovasc Med 2015;2:30.

6. Wendelboe AM, Raskob GE. Global Burden of Thrombosis: Epidemiologic Aspects. Circ Res 2016;118:1340-7.

7. Dang YP, Chen YF, Li YQ, et al. Developments of Anticoagulants and New Agents with Anti-Coagulant Effects in Deep Vein Thrombosis. Mini Rev Med Chem 2017;17:338-50.

8. Harmos S, Wang TK, Hawkins S, et al. Unprovoked DVT, the clot thickens. N Z Med J 2016;129:91-4.

9. Comerota AJ, Paolini D. Treatment of acute iliofemoral deep venous thrombosis: a strategy of thrombus removal. Eur J Vasc Endovasc Surg 2007;33:351-60; discussion 361-2.

10. Liew A, Douketis J. Catheter-directed thrombolysis for extensive iliofemoral deep vein thrombosis: review of literature and ongoing trials. Expert Rev Cardiovasc Ther 2016;14:189-200.

11. Lichtenberg MK, Jalaie H. Recanalization techniques for venous outflow obstruction. Dtsch Med Wochenschr 2016;141:1736-9. 
12. Cui YF, Fu YF, Liu HT, et al. Combined catheterdirected thrombolysis and iliac vein recanalization for iliac vein compression syndrome with secondary acute deep vein thrombosis: effectiveness and long-term outcome. Int Angiol 2016;35:40-6.

13. Vedantham S, Goldhaber SZ, Julian JA, et al. Pharmacomechanical Catheter-Directed Thrombolysis for Deep-Vein Thrombosis. N Engl J Med 2017;377:2240-52.

14. Han D, ó Hartaigh B, Lee JH, et al. Impact of D-Dimer for Prediction of Incident Occult Cancer in Patients with Unprovoked Venous Thromboembolism. PLoS One 2016;11:e0153514.

15. Prell J, Rachinger J, Smaczny R, et al. D-dimer plasma level: a reliable marker for venous thromboembolism after elective craniotomy. J Neurosurg 2013;119:1340-6.

16. Van de Werf F, Vanhaecke J, de Geest H, et al. Coronary thrombolysis with recombinant single-chain urokinasetype plasminogen activator in patients with acute myocardial infarction. Circulation 1986;74:1066-70.

17. Murray V, Norrving B, Sandercock PA, et al. The molecular basis of thrombolysis and its clinical application in stroke. J Intern Med 2010;267:191-208.

Cite this article as: Liu M, Zhang F. Administration routes affect thrombolytic effect of catheter-directed thrombolysis with pro-urokinase in treating deep vein thrombosis. Ann Transl Med 2018;6(16):322. doi: 10.21037/atm.2018.08.01
18. Yu YS, Zhou YR, Lu JS, et al. The construction and characterization of human pro-urokinase mutant. Sheng Wu Gong Cheng Xue Bao 2005;21:573-8.

19. Hawthorne C, Piper I. Monitoring of intracranial pressure in patients with traumatic brain injury. Front Neurol 2014;5:121.

20. Kesselman A, Kuo WT. Catheter-Directed Therapy for Acute Submassive Pulmonary Embolism: Summary of Current Evidence and Protocols. Tech Vasc Interv Radiol 2017;20:193-6.

21. Gao S, Zhu Q, Dong X, et al. Guided longer pulses from a diagnostic ultrasound and intraclot microbubble enhanced catheter-directed thrombolysis in vivo. J Thromb Thrombolysis 2017;44:48-56.

22. Wang CB, Hu HS, Liu YM. Animal study in combining targeted-microbubbles with low-frequency ultrasound for the treatment of ischemic stroke. Sichuan Da Xue Xue Bao Yi Xue Ban 2012;43:864-7.

23. Hsu PJ, Chen CH, Yeh SJ, et al. High Plasma D-Dimer Indicates Unfavorable Outcome of Acute Ischemic Stroke Patients Receiving Intravenous Thrombolysis. Cerebrovasc Dis 2016;42:117-21. 
Supplementary

Table S1 Body weight difference in 6 experimental groups

\begin{tabular}{|c|c|c|c|c|}
\hline Group & Number & Weight (kg) & $\mathrm{F}$ & $P$ \\
\hline Control & 6 & $2.933 \pm 0.242$ & & \\
\hline A & 6 & $2.983 \pm 0.214$ & & \\
\hline B & 6 & $2.933 \pm 0.250$ & & \\
\hline C & 6 & $2.933 \pm 0.207$ & 0.101 & 0.996 \\
\hline D & 6 & $2.900 \pm 0.179$ & & \\
\hline E & 6 & $2.917 \pm 0.204$ & & \\
\hline $\mathrm{F}$ & 6 & $2.900 \pm 0.228$ & & \\
\hline
\end{tabular}

Weight $(\mathrm{kg})$ are given as mean $\pm \mathrm{SD}$. Group A: systemic thrombolysis with intravenous injection; group B: CDT with pump infusion; group C: CDT with pulse injection; group D: CDT with combination of pump infusion and pulse injection; group E: catheter-directed aspiration thrombectomy; group F: mechanical thrombectomy. CDT, catheter-directed thrombolysis. 